\title{
Radiological Correlates of Raised Intracranial Pressure in Children: A Review
}

\author{
Saeed Kayhanian ${ }^{1}$, Adam M. H. Young ${ }^{2 *}$, Rory J. Piper ${ }^{2}$, Joseph Donnelly ${ }^{2}$, \\ Daniel Scoffings ${ }^{3}$, Matthew R. Garnett ${ }^{2}$, Helen M. Fernandes' ${ }^{2}$, Peter Smielewski², \\ Marek Czosnyka' ${ }^{2}$, Peter J. Hutchinson ${ }^{2}$ and Shruti Agrawal ${ }^{4}$ \\ 'School of Clinical Medicine, Addenbrooke's Hospital, University of Cambridge, Cambridge, United Kingdom, \\ ${ }^{2}$ Division of Academic Neurosurgery, Department of Clinical Neurosciences, Addenbrooke's Hospital, University of \\ Cambridge, Cambridge, United Kingdom, ${ }^{3}$ Department of Neuroradiology, Addenbrooke's Hospital, University of \\ Cambridge, Cambridge, United Kingdom, ${ }^{4}$ Department of Paediatric Intensive Care, Addenbrooke's Hospital, University of \\ Cambridge, Cambridge, United Kingdom
}

Radiological assessment of the head is a routine part of the management of traumatic brain injury. This assessment can help to determine the requirement for invasive intracranial pressure (ICP) monitoring. The radiological correlates of elevated ICP have been widely studied in adults but far fewer specific pediatric studies have been conducted. There is, however, growing evidence that there are important differences in the radiological presentations of elevated ICP between children and adults; a reflection of the anatomical and physiological differences, as well as a difference in the pathophysiology of brain injury in children. Here in, we review the radiological parameters that correspond with increased ICP in children that have been described in the literature. We then describe the future directions of this work and our recommendations in order to develop non-invasive and radiological markers of raised ICP in children.

Keywords: brain, injury, ONSD, transcranial Doppler ultrasound, intracranial pressure, basal cisterns

\section{INTRODUCTION}

Traumatic brain injury (TBI) in children remains a UK and worldwide public health concern. Early management of TBI aims to prevent secondary brain injury and invasive monitoring of intracranial pressure (ICP) plays an important role of the management of pediatric neurocritical patients (1).

The gold-standard for ICP measurement requires an invasive intraparenchymal monitor. Although generally regarded as safe, this procedure carries a small risk of hemorrhage, infection, and seizures (2-5). In patients with coagulopathies, invasive monitoring may be contra-indicated. Furthermore, in the global context of neurosurgery, a requirement for the expertise to insert such a device can result in delays in the implementation of guided medical therapy: some areas of the world are served by 1 neurosurgeon per 9 million patients (compared with the 1 per 80,000 in developed countries) (6). These issues have been brought to the fore by the results of a recent randomized-control trial in adults, which questioned the ostensible positive effect that invasive monitoring has on outcomes, stimulating debate as to whether invasive monitoring is over-utilized in current practice (7).

As such, an accurate and reproducible methodology for assessing raised ICP would be highly beneficial and allow for stratification of which patients would benefit from invasive monitoring. While radiological features are already recognized and used in clinical practice to alert to raised ICP 
(for example: midline shift, ventricular effacement, sulcal effacement, cistern effacement, and herniation), these features have not been extensively validated in a pediatric cohort.

Here in, we review the radiological parameters that correspond with increased ICP in children that have been described in the literature. We then describe the future directions of this work and our recommendations in order to develop non-invasive and radiological markers of raised ICP in children.

\section{STUDYING ICP IN CHILDREN}

The anatomical, physiological, and pathophysiological differences between children and adults mean that specific pediatric studies are essential in validating proposed radiological correlates of raised ICP.

In adults, intracranial hypertension (IH) is defined as an ICP that is persistently raised above $20 \mathrm{~mm} \mathrm{Hg}$ (8). In children, normal values are age dependent. While there is continued debate on age directed strategies, the consensus is that brief increases in ICP that return to normal in $<5$ min may be insignificant; however, sustained increases of $\geq 20 \mathrm{~mm} \mathrm{Hg}$ for $\geq 5 \mathrm{~min}$ should likely warrant treatment (9) (Figure 1).

Compared with adults, children may be more likely to develop diffuse brain swelling after TBI (10). This has been postulated to be because of immature or impaired autoregulation of cerebral perfusion pressure, an enhanced inflammatory response, and increased blood-brain barrier permeability in the developing brain $(11,12)$.

Children have a lower mean arterial blood pressure. This means that if a child does develop intracranial hypertension, they may be more likely to have a critically decreased cerebral blood flow (CBF) and thus sustain a secondary ischemic injury (13). There is extensive debate on whether management of acute brain injury should be targeted by ICP thresholds, by CPP thresholds or both. Only a small number of pediatric studies have demonstrated CPP-directed intervention. In two studies, the lower limit of the scale that was used was $40 \mathrm{~mm} \mathrm{Hg}(14,15)$, and in two other studies, it was $45 \mathrm{~mm} \mathrm{Hg}(16,17)$.

Furthermore, the biomechanical differences between adult and children's skull in relation to the brain offer different levels of accommodation. In infants, the presence of fontanelles allows for buffering of raised ICP (18).

\section{IMAGING MODALITIES}

The imaging modalities that have been tested against ICP are computed tomography (CT), magnetic resonance imaging (MRI), and ultrasonography (US). CT is routinely performed in children with suspected IH and so parameters in this modality are of importance in terms of immediate clinical utility. However, the lack of ionizing radiation with MRI and US make these modalities attractive alternatives, given that children are more radiosensitive and have longer life-expectancies compared with adults (19). However, MRI is rendered difficult with an uncooperative and distressed child due to long acquisition times and may be contraindicated in major trauma with potential metal foreign bodies. Furthermore, while US is available at the bedside, avoiding the hazards of patient transfer, and is radiation-free, it is not routinely used for this purpose in the context of TBI and there is limited, albeit growing, experience in this field.

\section{RADIOLOGICAL PARAMETERS}

A multitude of radiological parameters have been examined in children within small cohorts.

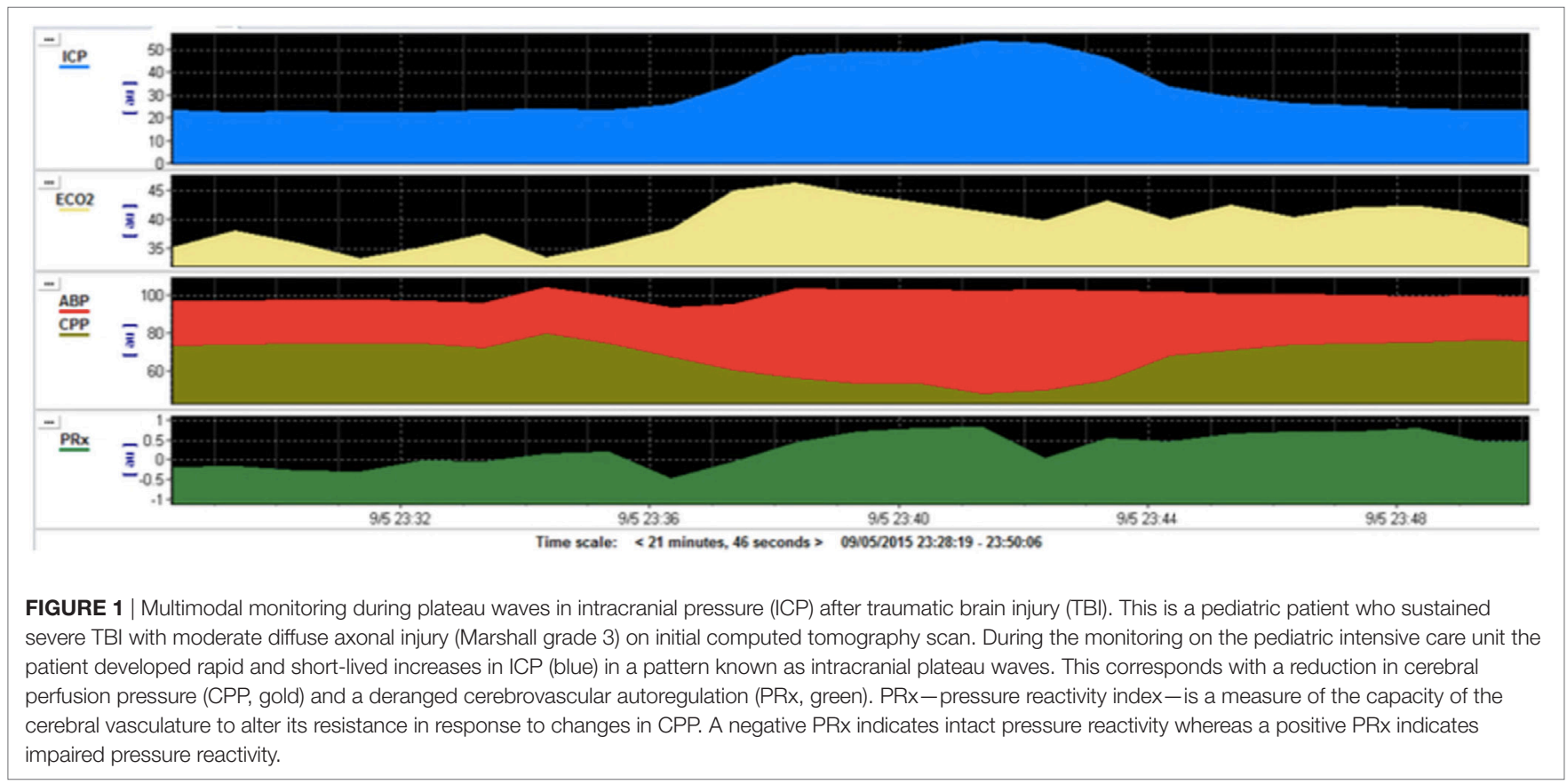




\section{Basal Cisterns}

The appearance of compressed or obliterated basal cisterns on CT images and its correlation to elevated ICP has been well studied in adult cohorts (20). Kouvarellis et al. have found that this correlation also holds true in children, with $75 \%$ of their cohort who had obliterated cisterns demonstrating at least one episode of elevated ICP on invasive monitoring (21). However, they also found that the presence of open cisterns does not necessarily correspond to normal ICP, with open cisterns having a positive predictive value of only $59 \%$ in detecting an ICP below $20 \mathrm{~mm} \mathrm{Hg}$. This is an important finding: an observation of patent cisterns in the presence of raised ICP can have significant implications for management of such patients. As such, a defined threshold of basal cistern compression in relation to raised ICP would be helpful to interpret this data. Moreover, the pathophysiology of this finding is interesting in itself. Given the close relationship with the pediatric brain with the inner table of the cranium, it is surprising that pathological ICP can be accommodated this efficiently, without displaying cardinal radiological signs of hypertension (Figure 2). Advances in understanding the mechanism of how the pediatric brain combats these fluctuations in pressure following injury may have multiple implications for CSF disorders.

\section{Optic Nerve Sheath Diameter}

The optic nerve sheath communicates with the subarachnoid space of the meninges and its diameter has been shown to widen in the context of elevated ICP (Figure 3) $(22,23)$. This phenomenon has been demonstrated to occur within minutes of acute changes in ICP and thus the ONSD poses an attractive target for non-invasive ICP monitoring (24).

Ultrasonography measurement of widened ONSD has been investigated as an indicator of elevated ICP in adults (25-27). In children, this correlation has also been demonstrated in a number of studies, with the cohort of 174 children in Padayachy et al. (largest to date) exhibiting a sensitivity of $80 \%$ in detecting ICP $\geq 20 \mathrm{~mm} \mathrm{Hg}$ (28). However, the reliable use of US may require technical expertise that is not widely available.

Only one study has explored the viability of ONSD in relation to CT values in pediatric cohorts (29). This study achieved a much-improved specificity (91\%) than similar studies in adult

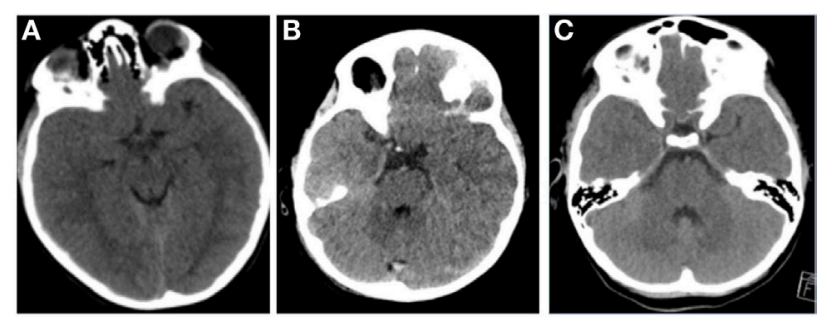

FIGURE 2 | Representative images of pediatric patients with raised intracranial pressure (ICP). (A) A patient with acute subdural hematoma (ASDH), opening ICP $32 \mathrm{~mm} \mathrm{Hg}$. (B) A patient with diffuse axonal injury, opening ICP $25 \mathrm{~mm} \mathrm{Hg}$. (C) A patient with ASDH, opening pressure $28 \mathrm{~mm}$ $\mathrm{Hg}$. All these pediatric patients demonstrate open basal cisterns, despite pathologically raised ICP. cohorts $[42 \%$, in one recent adult study (30)] to detect elevated ICP. It has been postulated that this difference may be as a result of children's brain parenchyma being intimate with the cranial vault, without the deep sulci that develop in late adolescence-meaning that changes in ONSD are subject to less inter-patient variation (28). This highlights the importance of deliberate pediatric studies in this field, with a variation in anatomy manifesting as a clear difference in neuroimaging parameters. The effectiveness of ONSD in a modality that is routinely acquired in current practice is of significant interest, although this being a single-center, small sized study means validation in larger cohorts is necessary.

A comparison of MRI to CT in measuring ONSD suggest that measurements should be in close agreement across both modalities and, indeed, Hirfanoglu et al. have recently demonstrated this potential correlation for children (31).

Thus, ONSD would seem a reliable parameter for evaluating ICP in children, being available in modalities of CT that is routinely used to diagnose TBI, and in US that reduces radiation exposure and minimizes time transferring patients from safety of the ICU. It is worth noting however, that the relationship between ONSD and raised ICP is dependent on establishing and validating threshold values above which ICP is considered elevated. The studies in children to date have been retrospective analyses that have used cutoff values that maximize the specificity and sensitivity of their measurements. It will be more difficult to adopt these cutoff values prospectively, given the interindividual variability in children of different ages in particular, but also of different genders and ethnicities $(32,33)$.

\section{Intracranial Elastance}

An emerging technique for measuring ICP using MRI is by using the concept of intracranial elastance. Elastance is defined as the ratio of change in pressure to change in volume, and an elastivity index has been determined for the brain over a range of ICPs (34). MRI analysis of CSF velocities and arterial, venous and CSF flow

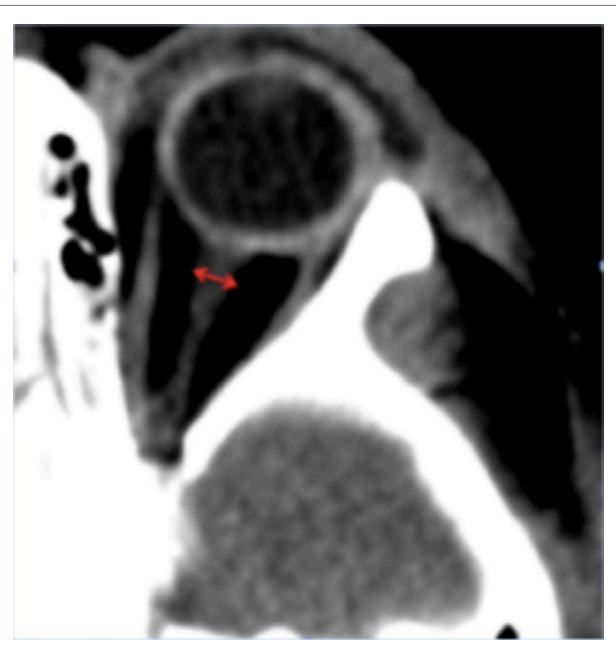

FIGURE 3 | Representative computed tomography image of a pediatric patient showing measurement of the ONSD. The ONSD is typically measured $3 \mathrm{~mm}$ behind the insertion of the optic nerve into the globe, perpendicular to the long axis of the optic nerve. 
volumes are used to calculate the small fluctuation in intracranial volume and pressure change during the cardiac cycle, which is then related to ICP using the known relationship between ICP and elastance (35).

There have been some studies to assess this model in adults but to date the only application in pediatric cohorts comes from Muehlmann et al. who found a positive correlation (Spearman $\rho=0.64, p<0.01$ ) between shunt opening pressure and MR-ICP in 15 children with hydrocephalus (36). Based on these observations there is reason to suspect that there is a potential role for intracranial elastance measurements in pediatric TBI patients. The technique is likely to be too cumbersome and time-consuming to provide rapid diagnosis and aid with decision-making criteria on therapy. However, there is a wide scope to use the modality to gain a greater understanding of the pathophysiology of intracranial hypertension following TBI and gain an insight into potential therapy.

\section{Cerebral Blood Flow}

Measurements of CBF can be studied using two modalities, transcranial Doppler ultrasound (TCD) magnetic resonance angiography (MRA). To date, only TCD has been used in the context of pediatric TBI.

Measurements of CBF by TCD rely on the observed physiological phenomenon that elevated ICP leads to a greater reduction in diastolic flow velocity than systolic flow velocity (37). This relationship is exploited by calculating the Gosling pulsatility index (PI), defined as the difference between systolic and diastolic flow velocities, divided by the mean flow velocity (37). The results of PI correlation to ICP in adults have shown limited utility, with numerous studies concluding that the relationship may only be reliable at extreme values of $\operatorname{ICP}(38,39)$. However, in children, this relationship may be of more value. Notably, the findings of O'Brien et al. suggest an extremely good correlation in the very early stages postinjury, with ICP $\geq 20 \mathrm{~mm} \mathrm{Hg}$ being predicted with $100 \%$ sensitivity and $82 \%$ specificity (40). This relationship does not seem to hold true more than $24 \mathrm{~h}$ postinjury, but this may still render TCD a valuable tool in screening which patients require invasive monitoring in monitoring for secondary brain injury (41).

Although MRA has not been demonstrated in pediatric TBI, the technique has been used in pediatric hydrocephalus patients. Measurement $\mathrm{CBF}$ at the level of the internal carotid artery and basilar artery were performed with the conclusion of only a moderate correlation $(r=-0.55)$ with raised ICP (42). However, significant reduction in $\mathrm{CBF}$ would be expected to require severe elevation in ICP. The study was performed in infants (age range $=1$ day to 7 months old) who were young enough to have open fontanelles. This makes it more likely, therefore, that these patients would have been able to tolerate significant rises in ICP before exhibiting any clinical signs. Indeed, Bateman, failed to

\section{REFERENCES}

1. Young AMH, Donnelly J, Czosnyka M, Jalloh I, Liu X, Aries MJ, et al. Continuous multimodality monitoring in children after traumatic brain injury-preliminaryexperience.PLoSOne(2016) 11(3):e0148817.doi:10.1371/ journal.pone.0148817 reproduce this correlation in a cohort of older children (mean age $=8 \pm 5$ ) (43). As such, given the complexity of the analysis and the time delay in image acquisition and analysis, MRA is unlikely to provide parameters that would be clinically useful in pediatric TBI.

\section{FUTURE DIRECTIONS}

A growing body of evidence is demonstrating some potentially beneficial modalities for using radiological parameters to guide therapy in pediatric TBI. Early work has already identified some thresholds to improve both sensitivity and specificity of such radiological markers.

There are existing radiological classification systems in adults, such as Marshall or Rotterdam scores, that have shown value in correlating radiological evidence to predict outcome $(44,45)$. While some of these have been validated in children there is scope to refine this to better suit the pathophysiology of pediatric TBI (46). A study combing the outlined measurements above would evaluate whether they serve to direct care more efficiently.

A validation of the radiological parameters of raised ICP on CT imaging would be of the most immediate clinical value, given this modality's widespread use in current practice. Currently, the parameters from adult studies are assumed as valid-an assumption that has already been questioned, as discussed above. Moreover, given the possible age-related differences (e.g., as a result of open fontanelles or changes in skull compliance), it would be prudent for future studies to stratify their pediatric cohorts by age, rather than group children as one demographic as some previous studies have done. Given the number of potential variables involved a large, prospective study specific to children would allow for validation of the most suitable radiological markers.

\section{AUTHOR CONTRIBUTIONS}

All authors listed, have made a substantial, direct and intellectual contribution to the work, and approved it for publication.

\section{FUNDING}

We gratefully acknowledge financial support as follows. Research support: the Medical Research Council (MRC, grant nos. G0600986 ID79068 and G1002277 ID98489) and the National Institute for Health Research Biomedical Research Centre (NIHR BRC) Cambridge (Neuroscience Theme; Brain Injury and Repair Theme). Authors' support: Peter J. Hutchinson-NIHR Research Professorship, Academy of Medical Sciences/Health Foundation Senior Surgical Scientist Fellowship and NIHR Cambridge BRC. Joseph Donnelly is supported by a Woolf Fisher Scholarship. MC-NIHR BRC.

2. Hoefnagel D, Dammers R, Ter Laak-Poort MP, Avezaat CJJ. Risk factors for infections related to external ventricular drainage. Acta Neurochir (Wien) (2008) 150(3):209-14. doi:10.1007/s00701-007-1458-9

3. Dasic D, Hanna SJ, Bojanic S, Kerr RSC. External ventricular drain infection: the effect of a strict protocol on infection rates and a review of the literature. Br J Neurosurg (2006) 20(5):296-300. doi:10.1080/02688690600999901 
4. Anderson RC, Kan P, Klimo P, Brockmeyer DL, Walker ML, Kestle JR. Complications of intracranial pressure monitoring in children with head trauma. J Neurosurg (2004) 101(1 Suppl):53-8. doi:10.3171/ ped.2004.101.2.0053

5. Binz DD, Toussaint LG, Friedman JA. Hemorrhagic complications of ventriculostomy placement: a meta-analysis. Neurocrit Care (2009) 10(2):253-6. doi:10.1007/s12028-009-9193-0

6. El Khamlichi A. Neurosurgery in Africa. Clin Neurosurg (2005) 52:214-7.

7. Chesnut RM, Temkin N, Carney N, Dikmen S, Rondina C, Videtta W, et al. A trial of intracranial-pressure monitoring in traumatic brain injury. $N$ Engl J Med (2012) 367(26):2471-81. doi:10.1056/NEJMoa1207363

8. Czosnyka M, Smielewski P, Kirkpatrick P, Laing RJ, Menon D, Pickard JD. Continuous assessment of the cerebral vasomotor reactivity in head injury. Neurosurgery (1997) 41(1):11-7. doi:10.1097/00006123-19970700000005

9. McLaughlin MR, Marion DW. Cerebral blood flow and vasoresponsivity within and around cerebral contusions. J Neurosurg (1996) 85(5):871-6. doi:10.3171/jns.1996.85.5.0871

10. Aldrich EF, Eisenberg HM, Saydjari C, Luerssen TG, Foulkes MA, Jane JA, et al. Diffuse brain swelling in severely head-injured children. A report from the NIH Traumatic Coma Data Bank. J Neurosurg (1992) 76(3):450-4. doi:10.3171/jns.1992.76.3.0450

11. Lang DA, Teasdale GM, Macpherson P, Lawrence A. Diffuse brain swelling after head injury: more often malignant in adults than children? J Neurosurg (1994) 80(4):675-80. doi:10.3171/jns.1994.80.4.0675

12. Adelson PD, Whalen MJ, Kochanek PM, Robichaud P, Carlos TM. Blood brain barrier permeability and acute inflammation in two models of traumatic brain injury in the immature rat: a preliminary report. Acta Neurochir Suppl (1998) 71:104-6.

13. Pinto PS, Meoded A, Poretti A, Tekes A, Huisman TA. The unique features of traumatic brain injury in children. Review of the characteristics of the pediatric skull and brain, mechanisms of trauma, patterns of injury, complications, and their imaging findings-part 2. J Neuroimaging (2012) 22(2):18-41. doi:10.1111/j.1552-6569.2011.00690.x

14. Kapapa T, König K, Pfister U, Sasse M, Woischneck D, Heissler H, et al. Head trauma in children, part 1: admission, diagnostics, and findings. J Child Neurol (2010) 25(2):146-56. doi:10.1177/0883073809332698

15. Stiefel MF, Udoetuk JD, Storm PB, Sutton LN, Kim H, Dominguez TE, et al. Brain tissue oxygen monitoring in pediatric patients with severe traumatic brain injury. J Neurosurg Pediatr (2006) 105(4):281-6. doi:10.3171/ ped.2006.105.4.281

16. Kochanek PM, Carney N, Adelson PD, Ashwal S, Bell MJB, Al S, et al. Guidelines for the acute medical management of severe traumatic brain injury in infants, children, and adolescents-second edition. Pediatr Crit Care Med (2012) 13:S1-2. doi:10.1097/PCC.0b013e31823f435c

17. Figaji AA, Zwane E, Thompson C, Fieggen AG, Argent AC, Le Roux PD, et al. Brain tissue oxygen tension monitoring in pediatric severe traumatic brain injury. Childs Nerv Syst (2009) 25(10):1325-33. doi:10.1007/s00381009-0822-x

18. Margulies SS, Thibault KL. Infant skull and suture properties: measurements and implications for mechanisms of pediatric brain injury. J Biomech Eng (2000) 122(4):364-71. doi:10.1115/1.1287160

19. Pauwels EKJ, Bourguignon MH. Radiation dose features and solid cancer induction in pediatric computed tomography. Med Princ Pract (2012) 21(6): 508-15. doi:10.1159/000337404

20. Toutant SM, Klauber MR, Marshall LF, Toole BM, Bowers SA, Seelig JM, et al. Absent or compressed basal cisterns on first CT scan: ominous predictors of outcome in severe head injury. J Neurosurg (1984) 61(4):691-4. doi:10.3171/ jns.1984.61.4.0691

21. Kouvarellis AJ, Rohlwink UK, Sood V, Van Breda D, Gowen MJ, Figaji AA. The relationship between basal cisterns on CT and time-linked intracranial pressure in paediatric head injury. Childs Nerv Syst (2011) 27(7):1139-44. doi:10.1007/s00381-011-1464-3

22. Launey Y, Nesseler N, Le Maguet P, Mallédant Y, Seguin P. Effect of osmotherapy on optic nerve sheath diameter in patients with increased intracranial pressure. J Neurotrauma (2014) 31(10):984-8. doi:10.1089/neu.2012.2829

23. Helmke K, Hansen HC. Fundamentals of transorbital sonographic evaluation of optic nerve sheath expansion under intracranial hypertension.
I. Experimental study. Pediatr Radiol (1996) 26(10):701-5. doi:10.1007/ BF01383383

24. Hansen H-C, Helmke K. Validation of the optic nerve sheath response to changing cerebrospinal fluid pressure: ultrasound findings during intrathecal infusion tests. J Neurosurg (1997) 87(1):34-40. doi:10.3171/jns.1997.87.1.0034

25. MORETTI R, PIZZI B. Ultrasonography of the optic nerve in neurocritically ill patients. Acta Anaesthesiol Scand (2011) 55(6):644-52. doi:10.1111/j. 1399-6576.2011.02432.x

26. Dubourg J, Javouhey E, Geeraerts T, Messerer M, Kassai B. Ultrasonography of optic nerve sheath diameter for detection of raised intracranial pressure: a systematic review and meta-analysis. Intensive Care Med (2011) 37(7): 1059-68. doi:10.1007/s00134-011-2224-2

27. Robba C, Cardim D, Tajsic T, Pietersen J, Bulman M, Donnelly J, et al. Ultrasound non-invasive measurement of intracranial pressure in neurointensive care: a prospective observational study. PLoS Med (2017) 14(7):e1002356. doi:10.1371/journal.pmed.1002356

28. Padayachy LC, Padayachy V, Galal U, Pollock T, Fieggen AG. The relationship between transorbital ultrasound measurement of the optic nerve sheath diameter (ONSD) and invasively measured ICP in children. Childs Nerv Syst (2016) 32(10):1779-85. doi:10.1007/s00381-016-3068-4

29. Young AM, Guilfoyle MR, Donnelly J, Scoffings D, Fernandes H, Garnett M, et al. Correlating optic nerve sheath diameter with opening intracranial pressure in pediatric traumatic brain injury. Pediatr Res (2017) 81(3):443-7. doi:10.1038/pr.2016.165

30. Sekhon MS, Griesdale DE, Robba C, McGlashan N, Needham E, Walland K, et al. Optic nerve sheath diameter on computed tomography is correlated with simultaneously measured intracranial pressure in patients with severe traumatic brain injury. Intensive Care Med (2014) 40(9):1267-74. doi:10.1007/ s00134-014-3392-7

31. Hirfanoglu T, Aydin K, Serdaroglu A, Havali C. Novel magnetic resonance imaging findings in children with intracranial hypertension. Pediatr Neurol (2015) 53(2):151-6. doi:10.1016/j.pediatrneurol.2015.03.028

32. Shofty B, Ben-Sira L, Constantini S, Freedman S, Kesler A. Optic nerve sheath diameter on MR imaging: establishment of norms and comparison of pediatric patients with idiopathic intracranial hypertension with healthy controls. AJNR Am J Neuroradiol (2012) 33(2):366-9. doi:10.3174/ajnr.A2779

33. Wang L, Feng L, Yao Y, Wang Y, Chen Y, Feng J, et al. Optimal optic nerve sheath diameter threshold for the identification of elevated opening pressure on lumbar puncture in a Chinese population. PLoS One (2015) 10(2):e0117939. doi:10.1371/journal.pone.0117939

34. Marmarou A, Shulman K, LaMorgese J. Compartmental analysis of compliance and outflow resistance of the cerebrospinal fluid system. J Neurosurg (1975) 43(5):523-34. doi:10.3171/jns.1975.43.5.0523

35. Raksin PB, Alperin N, Sivaramakrishnan A, Surapaneni S, Lichtor T. Noninvasive intracranial compliance and pressure based on dynamic magnetic resonance imaging of blood flow and cerebrospinal fluid flow: review of principles, implementation, and other noninvasive approaches. Neurosurg Focus (2003) 14(4):e4. doi:10.3171/foc.2003.14.4.4

36. Muehlmann M, Koerte IK, Laubender RP, Steffinger D, Lehner M, Peraud A, et al. Magnetic resonance-based estimation of intracranial pressure correlates with ventriculoperitoneal shunt valve opening pressure setting in children with hydrocephalus. Invest Radiol (2013) 48(7):543-7. doi:10.1097/ RLI.0b013e31828ad504

37. Gosling RG, King DH. Arterial assessment by Doppler-shift ultrasound. Proc R Soc Med (1974) 67(6 Pt 1):447-9.

38. Zweifel C, Czosnyka M, Carrera E, de Riva N, Pickard JD, Smielewski P. Reliability of the blood flow velocity pulsatility index for assessment of intracranial and cerebral perfusion pressures in head-injured patients. Neurosurgery (2012) 71(4):853-61. doi:10.1227/NEU.0b013e3182675b42

39. Behrens A, Lenfeldt N, Ambarki K, Malm J, Eklund A, Koskinen L-O. Transcranial Doppler Pulsatility Index: not an accurate method to assess intracranial pressure. Neurosurgery (2010) 66(6):1050-7. doi:10.1227/01. NEU.0000369519.35932.F2

40. O’Brien NF, Maa T, Reuter-Rice K. Noninvasive screening for intracranial hypertension in children with acute, severe traumatic brain injury. J Neurosurg Pediatr (2015) 16(4):420-5. doi:10.3171/2015.3.PEDS14521

41. LaRovere KL, O'Brien NF, Tasker RC. Current opinion and use of transcranial Doppler ultrasonography in traumatic brain injury in the pediatric 
intensive care unit. J Neurotrauma (2016) 33(23):2105-14. doi:10.1089/ neu.2015.4344

42. Leliefeld PH, Gooskens RH, Vincken KL, Ramos LM, van der Grond J, Tulleken CA, et al. Magnetic resonance imaging for quantitative flow measurement in infants with hydrocephalus: a prospective study. J Neurosurg Pediatr (2008) 2(3):163-70. doi:10.3171/PED/2008/2/9/163

43. Bateman GA. Cerebral blood flow and hydrocephalus. J Neurosurg Pediatr (2009) 3(3):244-244. doi:10.3171/2008.11.17594

44. Marshall LF, Marshall SB, Klauber MR, Van Berkum Clark M, Eisenberg H, Jane JA, et al. The diagnosis of head injury requires a classification based on computed axial tomography. J Neurotrauma (1992) 9(Suppl 1):S287-92.

45. Maas AIR, Hukkelhoven CWPM, Marshall LF, Steyerberg EW. Prediction of outcome in traumatic brain injury with computed tomographic characteristics: a comparison between the computed tomographic classification and combinations of computed tomographic predictors. Neurosurgery (2005) 57(6):1173-82. doi:10.1227/01.NEU.0000186013.63046.6B
46. Young AMH, Guilfoyle MR, Fernandes H, Garnett MR, Agrawal S, Hutchinson PJ. The application of adult traumatic brain injury models in a pediatric cohort. J Neurosurg Pediatr (2016) 18(5):558-64. doi:10.3171/ 2016.5.PEDS15427

Conflict of Interest Statement: The authors declare that the research was conducted in the absence of any commercial or financial relationships that could be construed as a potential conflict of interest.

Copyright (c) 2018 Kayhanian, Young, Piper, Donnelly, Scoffings, Garnett, Fernandes, Smielewski, Czosnyka, Hutchinson and Agrawal. This is an open-access article distributed under the terms of the Creative Commons Attribution License (CC BY). The use, distribution or reproduction in other forums is permitted, provided the original author(s) and the copyright owner are credited and that the original publication in this journal is cited, in accordance with accepted academic practice. No use, distribution or reproduction is permitted which does not comply with these terms. 